\title{
THE ADSORPTION AND INCORPORATION OF OXYGEN ON Cu(110) AND ITS REACTION WITH CARBON MONOXIDE
}

\author{
F.H.P.M. HABRAKEN and G.A. BOOTSMA \\ Van't Hoff Laboratory, State University of Utrecht, Padualaan $8,3584 \mathrm{CH}$ Utrecht, \\ The Netherlands
}

and

\author{
P. HOFMANN, S. HACHICHA and A.M. BRADSHAW \\ Fritz-Haber-Institut der Max-Planck-Gesellschaft, Faradayweg 4-6, D-1000 Beriln 33, \\ W. Germany
}

Received 23 March 1979; manuscript received in final form 29 June 1979

The initial interaction of oxygen with a $\mathrm{Cu}(110)$ surface occurs in two stages, characterised by $(2 \times 1)$ and $c(6 \times 2)$ overlayer structures and then a third stage where the $c(6 \times 2)$ structure remains but further oxygen uptake is registered only with ellipsometry. The first stage is nonactivated and is accompanied by a work function increase of $370-420 \mathrm{meV}$, depending on sample temperature. The subsequent appearance of the extra features associated with the $c(6 \times 2)$ structure in the second stage is accompanied by a decrease in work function of $\sim 100$ $\mathrm{meV}$ and is characterised by an apparent activation energy of $\sim 18 \mathrm{~kJ} \mathrm{~mol}^{-1}$. The adsorbed oxygen can be removed at all coverages by gas-phase $C O$. The reaction appears to follow Langmuir-Hinshelwood kinetics with an apparent activation energy of $\sim 25 \mathrm{~kJ} \mathrm{~mol}^{-1}$.

\section{Introduction}

The interaction of oxygen with the (110) surface of copper results in the initial formation of a $(2 \times 1)$ surface structure which is assumed to correspond to 0.5 monolayers [1-4]. Further oxygen exposure produces a $c(6 \times 2)$ structure [5]. The first stage of the interaction is known to be accompanied by an increase in work function. Thus Ertl and Küppers [6] have reported an increase of $0.2 \mathrm{eV}$ after an exposure of $6 \mathrm{~L}$ and Delchar [7] a maximum increase of $0.67 \mathrm{eV}$ after $2 \times 10^{3} \mathrm{~L}$ at room temperature. Delchar established that a change of $0.40 \mathrm{eV}$ had already been reached after about $100 \mathrm{~L}$. On the $(100)$ surface a similar reaction sequence has been observed. The formation of the initial $(\sqrt{ } 2 \times \sqrt{ } 2) R 45^{\circ}$ structure is accompanied by an increase in the work function as might be expected, but the formation of the subsequent $(\sqrt{2} \times 2 \sqrt{2}) R 45^{\circ}$ structure by a decrease $[8,9]$. This has been 
attributed to the incorporation of oxygen into the immediate sub-surface region. The starting point for the present investigation was to determine whether the formation of the $c(6 \times 2)$ structure on the $(110)$ surface is also indicative of incorporation phenomena.

We have studied the oxygen $/ \mathrm{Cu}(110)$ adsorption system with several different measuring techniques including LEED, AES, $\delta \phi$ and ellipsometry. The present paper concentrates on the $\delta \phi$ results and their correlation with LEED and ellipsometry data. A full account of the ellipsometric results for the first stage of the interaction will be published in a companion paper [4]. It has been found that the second stage of the interaction is indeed accompanied by a decrease in work function which is, however, not as large as in the case of the (100) surface. The change in the ellipsometric parameter $\Delta$ is found to be the only indicator of further reaction in the third stage at higher exposures: the work function and Auger signal do not change in this region.

We have also investigated the reaction of $\mathrm{CO}$ with oxygen adsorbed at various coverages. The results indicate that a Langmuir-Hinshelwood reaction mechanism operates with an apparent activation energy of $\sim 25 \mathrm{~kJ} \mathrm{~mol}^{-1}$. This value is in good agreement with the corresponding ellipsometric measurements reported in ref. [4] and with the LEED data of Ertl [10].

\section{Experimental}

The work function measurements were performed in a Varian UHV system (system I - Berlin) with a high gain self-compensating Kelvin probe. The chamber was pumped with an integrated ion and titanium sublimation pump unit. Further details are found in ref. [11]. The $\mathrm{Cu}(110)$ single crystal disc (Metals Research Ltd., 5N) was mechanically and electrochemically polished and mounted with $5 \mathrm{~N}$ copper wire such that it could be heated directly [8].

The ellipsometric measurements were performed in the system described in refs. [4] and [12] (system II - Utrecht). Here the crystal (Materials Research Corporation, $5 \mathrm{~N}$ ) was mounted in a stainless steel sample holder and heated indirectly. The wavelength of the light source for ellipsometry was $632.8 \mathrm{~nm}$ and the angle of incidence $69^{\circ} \pm 1^{\circ}$.

In both chambers the base pressure was in the low $10^{-10}$ Torr region and facilities were available for LEED and AES measurements. Cycles of argon ion bombardment $(600 \mathrm{eV}$ ) and annealing (up to $725 \mathrm{~K}$ ) were used to produce a clean surface as monitored with AES.

\section{The interaction of oxygen with $\mathrm{Cu}(110)$}

During the interaction of oxygen with a clean copper (110) surface at temperatures between 295 and $730 \mathrm{~K}$ three different stages could be distinguished. As an 


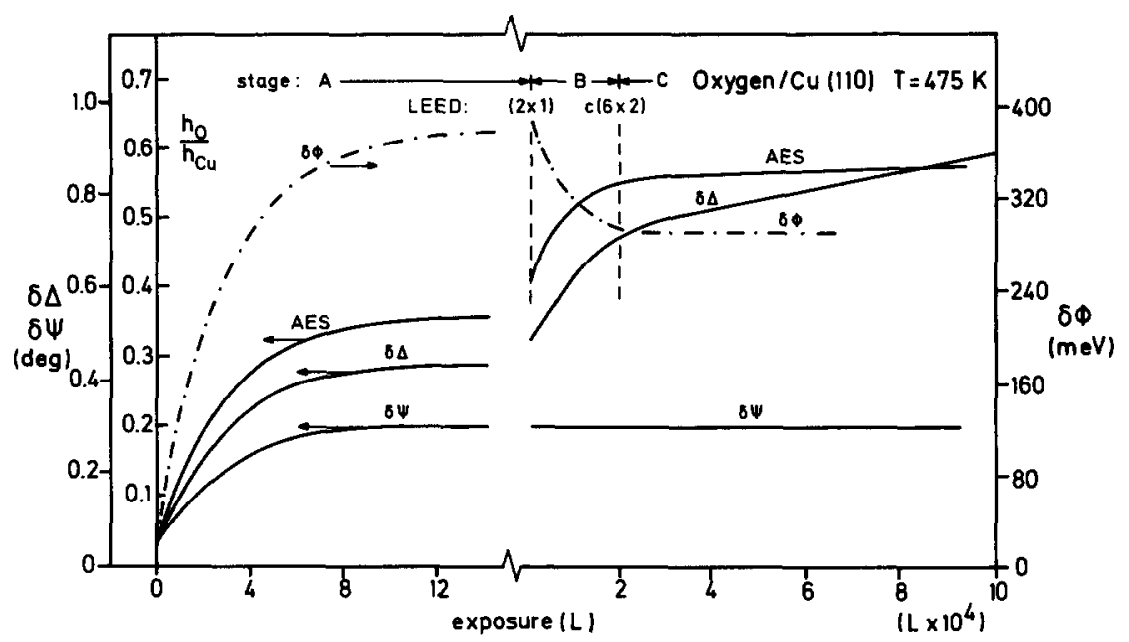

Fig. 1. Oxygen Auger signal (AES), $\delta \Delta, \delta \psi, \delta \phi$ and LEED patterns versus oxygen exposure at $475 \mathrm{~K}$. The plane of incidence of the light for ellipsometry was parallel to the [110] direction (i.e. $\Omega=0^{\circ}$ ).

example, fig. 1 shows the response of the four techniques (AES, LEED, $\delta \phi$ and ellipsometry) to oxygen exposure at $475 \mathrm{~K}$. The three stages are discussed in more detail below.

\subsection{The low exposure region (stage A)}

In system I continuous measurements of the kinetics of the first stage up to the formation of the $(2 \times 1)$ structure were difficult to perform because of the low oxygen pressure required and the disturbing influence of the ion pump and ion gauge. Using the cooled titanium sublimation pump without the ion pump and only turning on the ion gauge at the beginning and end of the measurement it was, however, possible to obtain $\delta \phi(\mathrm{L})$ curves such as that shown in fig. 1. In general the work function increase reached a maximum after about $200 \mathrm{~L}$. At room temperature $\delta \phi_{\max }$ was $370 \pm 10 \mathrm{meV}$, but increased at higher temperatures (see

Table 1

Maximum increase $( \pm 10 \mathrm{meV})$ in work function in the first chemisorption stage as a function of crystal temperature

\begin{tabular}{llllll}
\hline$T(\mathrm{~K})$ & 295 & 475 & 570 & 625 & 715 \\
\hline$\delta \phi_{\max }(\mathrm{meV})$ & 370 & 380 & 395 & 405 & 420 \\
\hline
\end{tabular}


table 1). At all temperatures the maximum corresponded to the establishment of the $(2 \times 1)$ surface structure. The increase in $\delta \phi_{\max }$ was not caused by a larger uptake of oxygen: adsorption at $715 \mathrm{~K}\left(\delta \phi_{\max }=420 \mathrm{meV}\right)$ followed by reduction at 460 $\mathrm{K}$ (where $\delta \phi_{\max }$ would be $380 \mathrm{meV}$ ) resulted in a clean surface, as monitored by AES, with $\delta \phi=-360 \mathrm{meV}$.

If it is assumed that $\delta \phi$ is proportional to coverage in this exposure region [6] and that $\delta \phi_{\max }$ corresponds to half a monolayer of oxygen atoms (section 3.3), the initial sticking coefficient $s_{0}$ is 0.25 and is independent of temperature ${ }^{*}$. The value is the same as that found with AES during interrupted exposures but is slightly higher than that found with ellipsometry and AES in system II $\left(s_{0} \sim 0.17\right.$ [4]). The difference in $s_{0}$ is undoubtedly due to different pressure readings in the two systems, which are caused by differing sensitivities of the ion gauges towards oxygen as well as by different positions of sample and ion gauges relative to the pumps.

\subsection{The high exposure region (stages $B$ and $C$ )}

The higher exposures were carried out with oxygen pressures between $2 \times 10^{-5}$ and $2 \times 10^{-4}$ Torr. After $\delta \phi_{\max }$ and the concomitant $(2 \times 1)$ structure had been reached the work function decreased (fig. 1, stage B). The total decrease amounted to $\sim 85 \mathrm{meV}$ at all temperatures and further exposure, even up to $10^{5} \mathrm{~L}$ at $630 \mathrm{~K}$, produced no further change. Fig. 2 shows, however, that at higher temperatures the rate of decrease is higher. Interruption of the exposure at any $\delta \phi$ during stage $B$ by evacuating the chamber did not cause any further changes.

In LEED the background intensity first rose during stage $B$ and at higher exposures the extra features indicative of both the $c(6 \times 2)$ and $(2 \times 1)$ surface structures were visible (fig. 3). The half-order features then disappeared leaving sharp spots from the $c(6 \times 2)$ structure by the time no further change in $\phi$ was apparent. This I.EFD sequence is in agreement with earlier authors [1-3].

The ellipsometric results obtained in system II are shown in fig. 4. The exposures were carried out at pressures between $5 \times 10^{-5}$ and $4 \times 10^{-4}$ Torr. Under these conditions $\delta \Delta$ rose rapidly by $\sim 0.5^{\circ}$ (stage $\mathrm{A}$ ), less rapidly up to $\delta \Delta \sim 0.7^{\circ}$ (stage B) and thereafter linearly in stage $C$. No saturation effect was observed in stage $C$ in contrast to the $\delta \phi$ and AES measurements. The maximum $\delta \Delta$ attained in this study was $1.5^{\circ}$ at $515 \mathrm{~K}$. The rate of change of $\delta \Delta$ appeared to be proportional to the oxygen pressure. Two-zone measurements showed that the other ellipsometric parameter $\delta \psi$ rises in the low exposure region to $0.3^{\circ}$, thereafter remaining constant (at $0.3^{\circ} \pm 0.03^{\circ}$ ) in the high exposure region. This behaviour has also been

"In this paper the term "monolayer" indicates a surface concentration of $1.1 \times 10^{15}$ oxygen atoms $\mathrm{cm}^{-2}$ independent of whether they are chemisorbed or incorporated into the surface. 


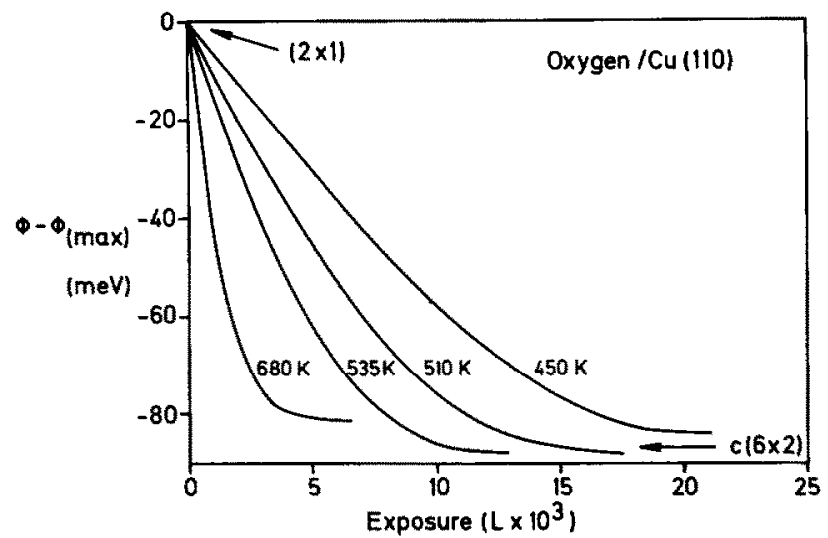

Fig. 2. Change in work function with respect to $\delta \phi_{\max }$ as a function of oxygen exposure.
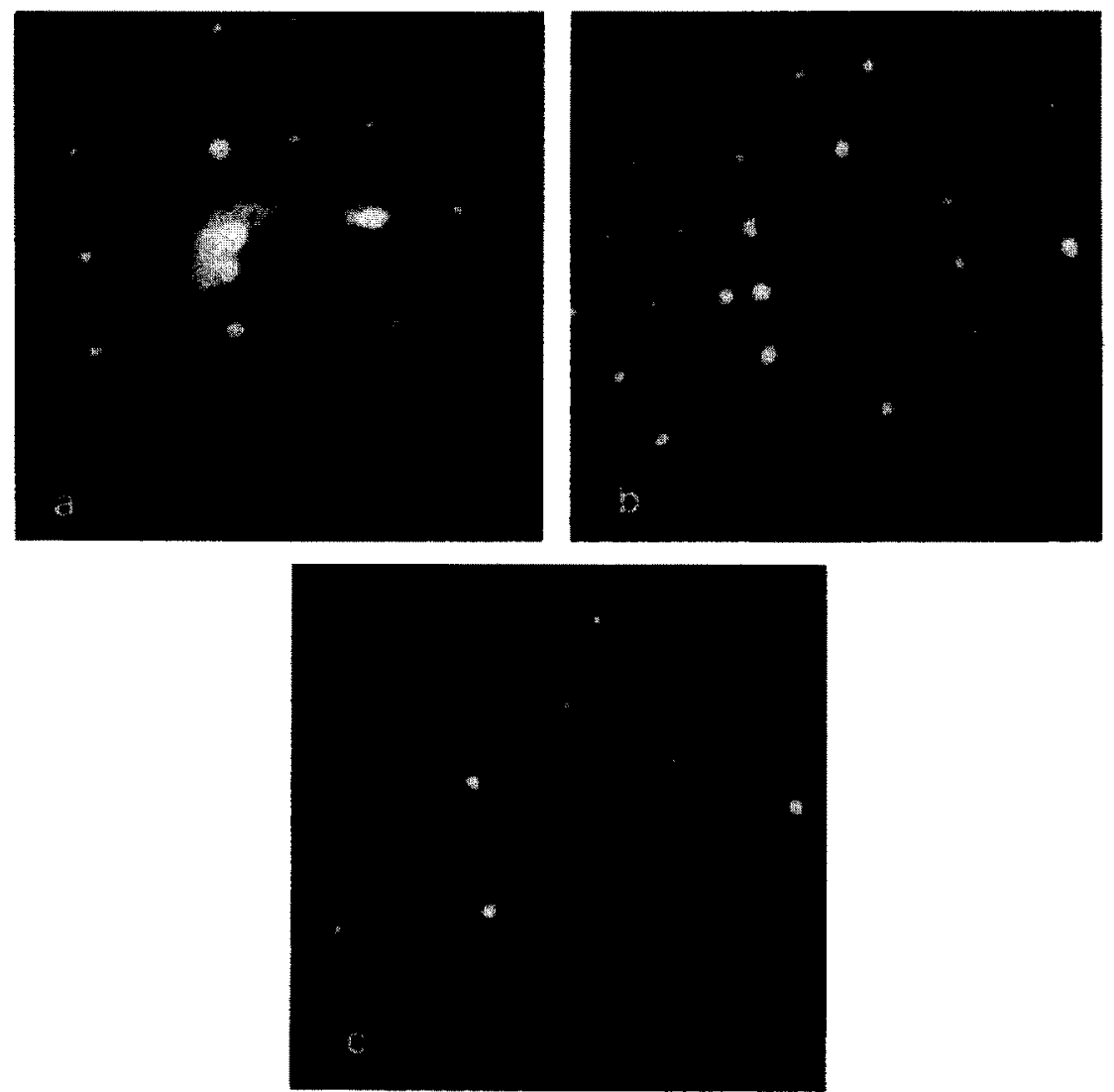

Fig. 3. LEED patterns in the second stage of the interaction: (a) $(2 \times 1)$ pattern $(90 \mathrm{eV})$; (b) domain structure $(70 \mathrm{eV}) ;(\mathrm{c}) \mathrm{c}(6 \times 2)$ pattern $(70 \mathrm{eV})$. 
found on the (111) surface [12]. No disturbing influence due to hot filaments was detected in these experiments.

At the higher pressures employed in stages $\mathrm{B}$ and $\mathrm{C}$ another gauge was used in system I and the vacuum chamber was isolated from the pumps with a poppet valve. Under these conditions the exposure scales appeared to be approximately equal in both systems. This could be estimated from the measurements of $\mathrm{CO}$ reaction probabilities described in section 4 below.

\subsection{Discussion}

From fig. 1 the initial interaction of oxygen with the (110) surface of copper can be divided phenomenologically into the three stages $\mathrm{A}, \mathrm{B}$ and $\mathrm{C}$ described above. The increase in work function up to 0.5 monolayers at low oxygen exposure indicates simple chemisorption without reconstruction or incorporation. This result is in agreement with a recent low-energy ion scattering study [13]. Analogous to the situation in the oxygen $/ \mathrm{Cu}(100)$ system [8] the reversal of the direction of the work function change in stage $B$ appears to indicate some degree of incorporation into the sub-surface region. A similar conclusion can be drawn from the ellipsometric measurements. The data reported here are comparable with those obtained from the $\mathrm{O}_{2} / \mathrm{Cu}(111)$ system [12]. There, the second stage was characterised by an increase in $\delta \Delta$, while $\delta \psi$ and the oxygen Auger signal remained constant, indicating oxygen uptake beneath the surface [12]. A ratio of 2.3 for the $\delta \Delta$ per $O$ atom adsorbed to $\delta \Delta$ per $O$ atom absorbed was observed. When this ratio of 2.3 is also assumed to hold for the $\mathrm{O}_{2} / \mathrm{Cu}(110)$ system, then after the uptake of the second half monolayer, the total $\delta \Delta$ should be about $0.7^{\circ}$. This value is reached in the region where the slopes in fig. 4 become constant, the $c(6 \times 2)$ pattern is fully

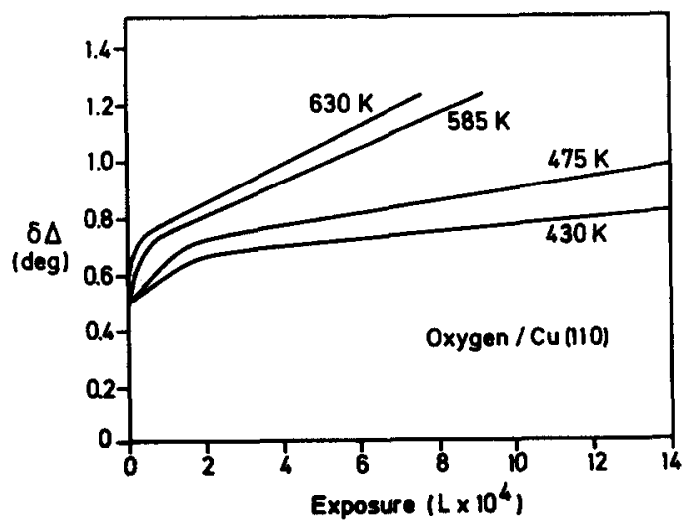

Fig. 4. The change in $\Delta$ as a function of exposure of oxygen to a clean and annealed $\mathrm{Cu}(110)$ surface. (Off-null irradiance measurements, with the plane of incidence parallel to the [110] direction.) 


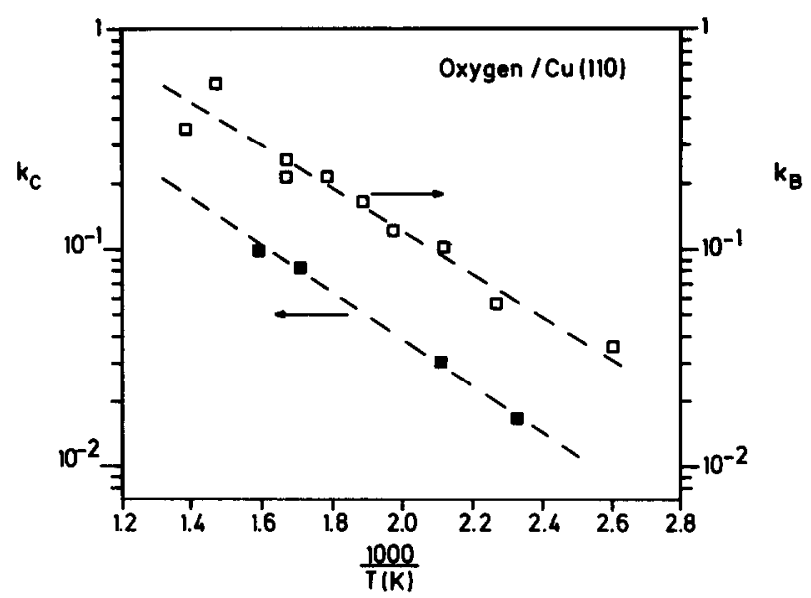

Fig. 5. Arrhenius plot of $\mathrm{d}(\delta \phi) / \mathrm{d} t$ at $\theta=0.5\left(k_{\mathrm{B}}\right)$ and $\mathrm{d}(\delta \Delta) / \mathrm{d} t$ in stage $\mathrm{C}\left(k_{\mathrm{C}}\right)$ (arbitrary units).

developed and the work function no longer changes (fig. 1). We tentatively ascribe this coverage to be the monolayer point $\theta=1$. Because AES appears to be less sensitive for this second half-monolayer (see fig. 1), it can be concluded that some oxygen atoms are already partly located beneath the surface, but still in the immediate surface region. In stage B the LEED pattern showed domains of $(2 \times 1)$ and $c(6 \times 2)$. This is an indication of a nucleation and growth process in this coverage region. Because oxygen in the gas phase is required for the conversion of $(2 \times 1)$ to $c(6 \times 2)$, a co-operative sorption process would occur, whereby the conversion is linked mechanistically to the dissociation of further oxygen molecules. A similar mechanism has been proposed for the $\mathrm{Cu}(100)$ surface [8]. If we assume that in stage B $\delta \Delta$ is also proportional to $\theta$, then the sticking coefficient at $\theta=0.5\left(\mathrm{~s}_{0.5}\right)$ may be calculated from fig. 2 and from the calibration with ellipsometry. At $T=$ $450 \mathrm{~K}$, for example, $s_{0.5}=6 \times 10^{-5}$. From the initial rate of decrease in $\phi$ at $\theta=0.5$ as a function of temperature (see e.g. fig. 2) it is possible to derive an apparent activation energy of $\sim 18 \mathrm{~kJ} \mathrm{~mol}^{-1}$ for the co-operative sorption process (fig. 5). Ertl [10] has obtained a value of $\sim 15 \mathrm{~kJ} \mathrm{~mol}^{-1}$ using the changes in intensity of the additional LEED features.

The above incorporation mechanism notwithstanding, it must be noted, however, that the reversal in work function change during stage $B$ is not as large as that occurring on the $\mathrm{Cu}(100)$ surface, where a net decrease results at sufficiently high exposures [8]. This may indicate that the $c(6 \times 2)$ structure is a co-incidence site lattice where certain oxygen atoms in the unit cell can penetrate somewhat further into the surface than is the case for the $(2 \times 1)$ structure. According to De Wit et al. [13] the most likely sites for the latter are the "long bridges". If in the $c(6 \times 2)$ structure some oxygen atoms are adsorbed in deeper $B_{5}$ sites [14] due to a re-distri- 
bution along the $[\overline{1} 10]$ direction, then a decrease in net work function might result.

In stage $C$ only $\Delta$ is found to vary. In fig. 5 the slopes from the linear parts of the curves in fig. 4 are thus plotted as well. For this stage of the interaction the apparent activation energy again appears to be $\sim 18 \mathrm{~kJ} \mathrm{~mol}^{-1}$. When the same calibration is used as for stage $B$, then the sticking coefficients are about an order of magnitude lower than $s_{0.5}$. The equality of the activation energies is, however, an indication that the same process is rate limiting in both stages. The proportionality of the rates to the oxygen pressure suggests strongly that this process is taking place at the surface and is in fact the dissociation of a physisorbed molecular precursor state. It should be remarked that when the precursor state is not fully accommodated the value of the activation encrgy obtained from the Arrhenius plots of fig. 5 is smaller than the actual value. Only variation of the gas temperature will give the real activation energy in an Arrhenius plot.

The bulk oxidation studies of Lawless and Gwathmey [15] indicate that $\mathrm{Cu}_{2} \mathrm{O}(110)$ will grow epitaxially on $\mathrm{Cu}(110)$ under the appropriate conditions of temperature and pressure. We have been unable to correlate the $(2 \times 1)$ or $c(6 \times 2)$ structures with particular oxidic configurations, neither in $\mathrm{Cu}_{2} \mathrm{O}$ nor in $\mathrm{CuO}$. Jardinier-Offergeld and Bouillon [16] have studied the kinetics of formation of oxide nuclei on a $\mathrm{Cu}(110)$ surface at oxygen pressures between $5 \times 10^{-5}-5 \times 10^{-4}$ Torr and at temperatures $770-970 \mathrm{~K}$ with a microgravimetric technique. If their results are extrapolated into our temperature range it would appear that in the present study the critical oxygen coverage at which nuclei formation begins was not reached.

\section{The oxidation of carbon monoxide by preadsorbed oxygen}

\subsection{Results}

Exposures of $\mathrm{CO}$ to surfaces with various oxygen coverages were carried out in system $\mathrm{I}$ in the pressure range $1 \times 10^{-4}-3.5 \times 10^{-3}$ Torr and at sample temperatures between 450 and $735 \mathrm{~K}$. During the $\mathrm{CO}$ exposures the ionisation gauge was switched off. The change in work function as a result of $\mathrm{CO}$ exposure, for cases where the initial oxygen coverage $\theta_{0}$ was about 0.5 , is depicted in fig. 6 . The initial value of $\delta \phi$ was obtained from the value of $\phi$ at the completion of the reduction when no further change occurred and the surface was clean as monitored with AES. Similar to the observations reported in section 3.1 the total $\delta \phi$ measured in this manner also increased with increasing temperature. From experiments at different $\theta_{0}(\leqslant 0.5)$ it could be shown that $\mathrm{d}(\delta \phi) / \mathrm{d}(p t)$ was only a function of $\theta$ and $T$ not of $\theta_{0}$ in this coverage region.

A typical $\delta \phi$ curve in an experiment where larger oxygen exposures were involved is depicted in fig. 7. Approximately $1 \mathrm{~h}$ after the interaction with oxygen 


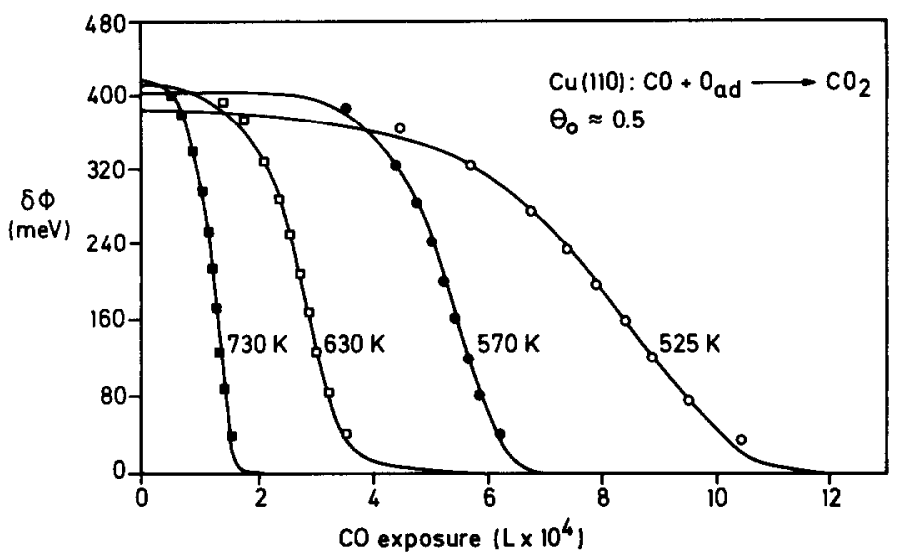

Fig. 6. Change in work function upon exposure of $\mathrm{CO}$ to a $\mathrm{Cu}(110)$ surface with an initial oxygen coverage $\theta_{0}=0.5, p_{\mathrm{CO}}=2 \times 10^{-5}-8 \times 10^{-5}$ Torr (curves). The symbols denote the integral of eq. (1) with (o) $k_{1}=6.4 \times 10^{-5}$; (•) $k_{1}=1.3 \times 10^{-4}$; (口) $k_{1}=1.75 \times 10^{-4}$; (口) $k_{1}=$ $3.5 \times 10^{-4}$, and $k_{2}=2 k_{1}\left(\right.$ all in $\left.\mathrm{L}^{-1}\right)$.

CO was admitted at $9 \times 10^{-4}$ Torr with the poppet valve closed. At first the work function increased, passed through a maximum, $\delta \phi_{\max }^{\prime}$, and then decreased, eventually reaching the value $( \pm 15 \mathrm{meV})$ of before the experiment. On pumping the $\mathrm{CO}$ away no further change in $\phi$ occurred. In all cases $\delta \phi_{\max }^{\prime}$ was found to be smaller

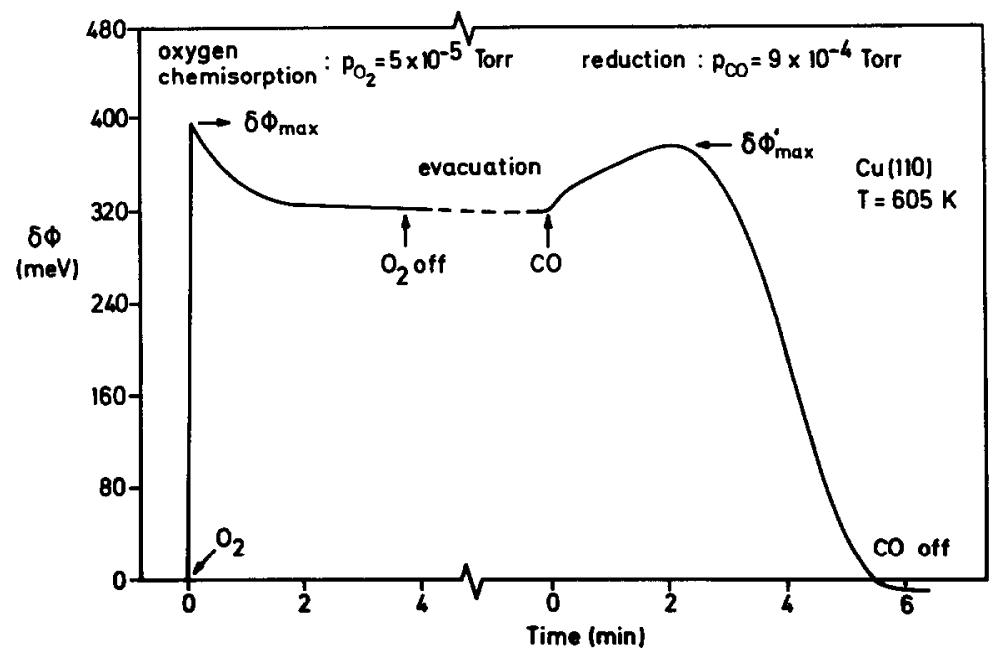

Fig. 7. Typical plot of $\delta \phi$ for the interaction with oxygen up to $\theta=1$ and subsequent reduction of a $\mathrm{Cu}(110)$ surface (see text). 


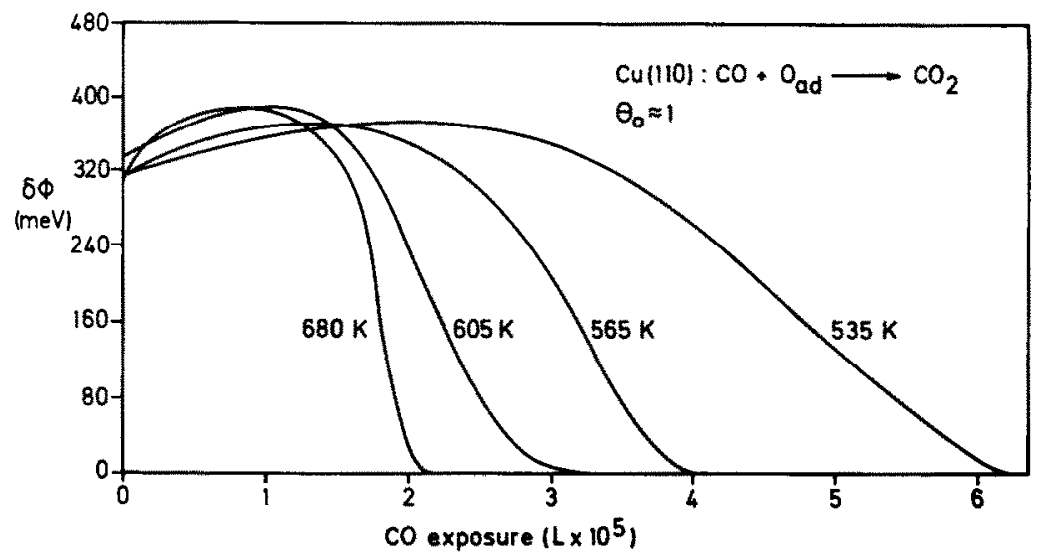

Fig. 8. Change in work function upon exposure of $C O$ to a $C u(110)$ surface with $\theta_{0}=1, p_{\mathrm{CO}}=$ $1 \times 10^{-4}-4 \times 10^{-3}$ Torr.

than $\delta \phi_{\max }$. Fig. 8 shows $\delta \phi$ as a function of $\mathrm{CO}$ exposure at different crystal temperatures. Here $\theta_{0}$ was about unity corresponding to the $c(6 \times 2)$ surface structure. It appeared that $\mathrm{d}(\delta \phi) / \mathrm{d} t$ was approximately proportional to pressure in both regions, indicating a reaction path not involving the dissociation of $\mathrm{CO}$.

Ellipsometric two-zone measurements (system II) of the reduction of a surface at higher oxygen coverages $\left(\delta \Delta \sim 1.0^{\circ}\right)$ are shown in fig. 9. Initially $\delta \Delta$ decreased

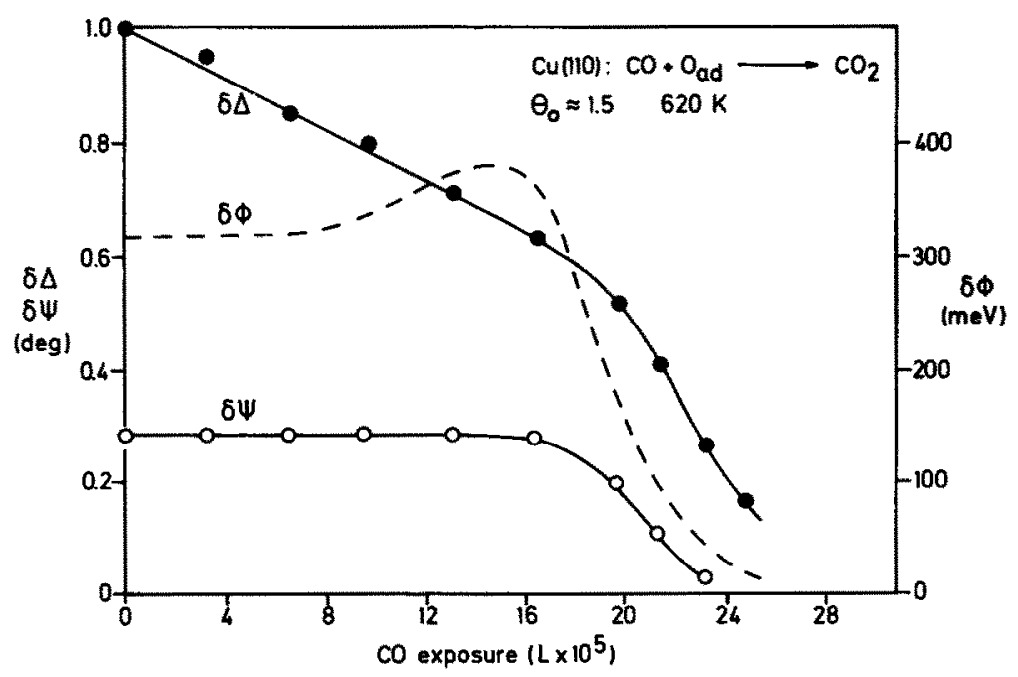

Fig. 9. Ellipsometric effects upon exposure of $\mathrm{CO}$ to a $\mathrm{Cu(110)}$ surface with an initial oxygen coverage equivalent to $\sim 1.5$ monolayer $\left(p_{\mathrm{CO}}=3 \times 10^{-4}\right.$ Torr). A corresponding $\delta \phi$ curve is also included. 
linearly down to $\sim 0.6^{\circ}$ after which a change of slope took place. The other ellipsometric parameter $\psi$ remained up to this point constant. A typical $\delta \phi$ curve has been included in this figure to show that the work function remains constant down to $\delta \Delta \sim 0.7^{\circ}-0.8^{\circ}$ (or $\theta \sim 1.0$ ).

\subsection{Discussion}

For the linear part of the $\delta \phi$ curves obtained with starting coverages smaller than or equal to half a monolayer, reaction probabilities have been calculated. These reaction probabilities (defined as the number of oxygen atoms removed per incident $\mathrm{CO}$ molecule) are depicted in an Arrhenius plot in fig. 10, together with the corresponding values obtained with ellipsometry in system II [4] (curve A). At all temperatures it has been assumed that $\delta \phi_{\max }$ corresponds to half a monolayer and that $\delta \phi$ is proportional to $\theta$. There is good agreement both for absolute values $\left(5 \times 10^{-5}\right.$ at $T=570 \mathrm{~K})$ and for the apparent activation energy $\left(\sim 25 \mathrm{~kJ} \mathrm{~mol}^{-1}\right)$. Similar curves have been observed for the removal of oxygen adsorbed on a $\operatorname{Ag}(110)$ surface [17]. There, however, the reaction appeared to proceed much more easily with an apparent activation energy of $\sim 9 \mathrm{~kJ} \mathrm{~mol}^{-1}$ and a reaction probability of $5 \times 10^{-3}$ at room temperature. The heat of adsorption of $\mathrm{CO}$ on $\mathrm{Cu}(110)$ is reported to be $55 \mathrm{~kJ} \mathrm{~mol}^{-1}$ [18]. Thus the equilibrium concentration of $\mathrm{CO}_{\mathrm{ad}}$ will vary as $\exp \left(55 \mathrm{~kJ} \mathrm{~mol}^{-1} / R T\right)$ and is expected to be very small in the range of temperatures and pressures used in the present investigation. For the effective activation energy for the reaction $\mathrm{CO}_{\mathrm{ad}}+\mathrm{O}_{\mathrm{ad}} \rightarrow \mathrm{CO}_{2}$ we therefore obtain $80 \mathrm{~kJ} \mathrm{~mol}^{-1}$, which is about equal to the value obtained for the same reaction on $\mathrm{Cu}(111)$ [12].

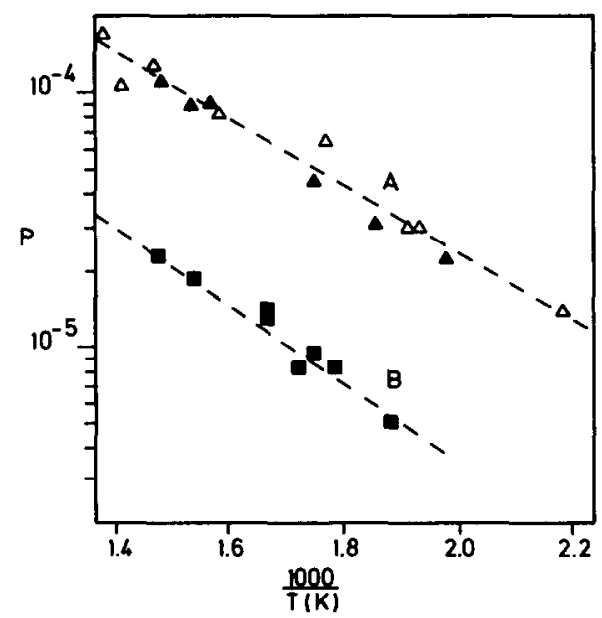

Fig. 10. Arrhenius plot of reaction probabilities $P$ for the reaction of $\mathrm{CO}$ with adsorbed oxygen. $(\Delta) \delta \phi$ system I, $\theta_{0}=0.5 ;(\Delta)$ ellipsometry system II, $\theta_{0}=0.5 ;(\bullet) \delta \phi$ system I, $\theta_{0}=1$. 
The curves of fig. 6 can be explained in the same way as the ellipsometric measurements of ref. [4]. Using the Langmuir-Hinshelwood mechanism and a onedimensional model for the reaction, the following rate equation has been obtained:

$\frac{\mathrm{d} \theta^{\prime}}{\mathrm{d}\left(p_{\mathrm{CO}} t\right)}=\left\{-2 k_{1} \theta^{\prime}-\frac{4\left(k_{2}-k_{1}\right) \theta^{\prime}\left(1-\theta^{\prime}\right)}{\left\{1-4 \theta^{\prime}\left(1-\theta^{\prime}\right)[1-\exp (\omega / R T)]\right\}^{1 / 2}+1}\right\}\left(1-\theta^{\prime}\right)$,

where $\theta^{\prime}=2 \theta ; k_{1}$ and $k_{2}$ are the different rate constants for reaction with "row" oxygen atoms and with "lone" oxygen atoms respectively $[17,4]$. For fitting to the curves of fig. 6 it is assumed that $k_{2}=2 k_{1}$ and that the interaction energy $\omega$ between adsorbed oxygen atoms in the [001] direction is $17 \mathrm{~kJ} \mathrm{~mol}^{-1}$. The points in fig. 6 represent the fits for the different $k_{1}$ values given in the caption.

Upon exposure of $\mathrm{CO}$ to a surface with an oxygen coverage larger than half a monolayer, first an increase in $\phi$ was observed (figs. 7 and 8). This is in agreement with the reversible character of the initial interaction previously observed by Ertl [10] for $\mathrm{Cu}(110)$ and in ref. [12] for $\mathrm{Cu}(111)$. Ertl [10] has observed with LEED that removal of oxygen from a surface with the $c(6 \times 2)$ pattern proceeds via the formation of a domain structure. Thus at coverages between those associated with the fully developed $c(6 \times 2)$ and $(2 \times 1)$ patterns, there exist domains with a $(2 \times 1)$ and domains with a $c(6 \times 2)$ structure on the surface. At first regions on the surface are converted from the $c(6 \times 2)$ pattern to the $(2 \times 1)$ pattern, and this causes the work function to increase, exactly opposite to the formation of the $c(6 \times 2)$ structure from the $(2 \times 1)$ structure during the initial interaction with oxygen. If all the $c(6 \times 2)$ regions are first converted into $(2 \times 1)$ regions, then $\delta \phi_{\max }^{\prime}$ should be equal to $\delta \phi_{\max }$. The measurements show, however, that $\delta \phi_{\max }^{\prime}$ is about $15 \mathrm{meV}$ smaller than $\delta \phi_{\max }$. The $\delta \phi$ of a domain with the $(2 \times 1)$ structure is about $400 \mathrm{meV}$ at these temperatures, that of a $c(6 \times 2)$ domain about $320 \mathrm{meV}$. Assuming that the measured work function is a surface-area-weighted average over both structures, then in the maximum $\left(\delta \phi_{\max }^{\prime}\right)$ at most a relative surface area of 0.2 is covered with $\mathrm{c}(6 \times 2)$ domains. In region $\mathrm{B}$ it is clearly not possible to calculate a well-defined reaction probability. We therefore first turn our attention to the low coverage region of the curves in fig. 8.

In fig. 10 are shown the reaction probabilities from the linear parts $(\delta \phi \sim 100$ $\mathrm{mV}$ ) of curves such as in fig. 8 plotted semi-logarithmically against $1 / T$ (curve B). It has been assumed that in the low coverage region $\delta \phi$ is proportional to the coverage and that the values of table 1 (section 3.1) for half a monolayer may be used for the purposes of calibration. Curve B is approximately parallel to curve A ( $E_{\text {act }} \sim$ $25 \mathrm{~kJ} \mathrm{~mol}^{-1}$ ) but the reaction probabilities are a factor six lower. This indicates that the rate controlling step of the reaction is also the same when high starting coverages are used, but that a different pre-exponential factor is involved. Measurements made between $\theta=0.5$ and 1.0 with $\theta_{0}=1$ (assuming proportionality to coverage and complete conversion of $\mathrm{c}(6 \times 2)$ to $(2 \times 1)$ - see above) gave reaction probabilities with values lying on curve $B$. With $0.5<\theta_{0}<1.0$ reaction probabili- 
ties between A and B were obtained. Although, for the reasons given above, these latter measurements are less well-defined, they do further indicate that the preexponential factor depends on the starting coverage not on the coverage at which the reaction is occurring. For $\theta_{0}>0.5$ it is possible that small domains with the $c(6 \times 2)$ structure inhibit the growth of islands of bare surface and thus lower the reaction probability. Inspection of figs. 6 and 8 reveals that the initial reaction rate is virtually zero at $\theta_{0}=0.5$ but non-zero at $\theta_{0} \approx 1.0$. This indicates that the $c(6 \times 2)$ pattern is characteristic of a surface which is structurally altered in such a way that CO can adsorb and react more easily than at $\theta=0.5$.

Finally we discuss the ellipsometric studies of $\mathrm{CO}$ oxidation. Taking into account a different sensitivity for surface and sub-surface oxygen [12], it is possible to explain the ellipsometric data of fig. 9 in a similar way. The initial oxygen coverage is assumed to be 1.5 monolayers and the oxygen atoms are partly located on the surface and partly below it (see section 3.3). The changes in $\delta \Delta, \delta \psi$ and $\delta \phi$ here are also opposite to the changes induced by oxygen interaction. Initially the net effect is the removal of oxygen atoms from beneath the surface: $\delta \Delta$ changes linearly and it thus appears that the reaction rate also remains constant. Even though the reaction takes place on the surface, empty sites are quickly filled up by oxygen atoms from beneath the surface. The removal of $(2 \times 1)$ oxygen starts at $\delta \Delta \sim 0.6^{\circ}$, when $\delta \psi$ begins to decrease. The $\delta \Delta$ for a surface with the $(2 \times 1)$ structure is about $0.5^{\circ}$; thus at $\delta \Delta \sim 0.6^{\circ}$ not all the $c(6 \times 2)$ domains with $\theta>1$ have disappeared. About the same ratio of 2.3 for the sensitivities for surface and subsurface oxygen seems to hold here. The ratio cannot be determined accurately because in the last stage of the reduction two or three processes in different domains are probably measured simultaneously.

\section{Acknowledgements}

The investigations in Utrecht were supported by the Netherlands Foundation of Chemical Research (SON) with financial aid from the Netherlands Organisation for the Advancement of Pure Research (ZWO). The investigations in Berlin were supported in part by the Deutsche Forschungsgemeinschaft.

\section{References}

[1] G. Ertl, Surface Sci. 6 (1967) 208.

[2] G.W. Simmons, D.F. Mitchell and K.R. Lawless, Surface Sci. 8 (1967) 130.

[3] A. Oustry, L. Lafourcade and A. Escaut, Surface Sci. 40 (1973) 545.

[4] F.H.P.M. Habraken and G.A. Bootsma, Surface Sci. 87 (1979) 333.

[5] In the matrix notation this structure is described by $\left[\begin{array}{ll}3 & 1 \\ 0 & 2\end{array}\right]$.

[6] G. Ertl and J. Küppers, Surface Sci. 24 (1971) 104.

[7] T.A. Delchar, Surface Sci. 27 (1971) 11. 
[8] P. Hofmann, R. Unwin, W. Wyrobisch and A.M. Brad shaw, Surface Sci. 72 (1978) 635.

[9] C. Benndorf, B. Egert, G. Keller, H. Seidel and F. Thieme, J. Phys. Chem. Solids, in press.

[10] G. Ertl, Surface Sci. 7 (1967) 309;

G. Ertl, in: Molecular Processes on Solid Surfaces, Eds. E. Drauglis, R.D. Gretz and R.I. Jaffee (McGraw-Hill, New York, 1969) p. 147.

[11] P. Hofmann, Thesis, Tech. Univ. Munich (1979).

[12] F.H.P.M. Habraken, E.Ph. Kieffer and G.A. Bootsma, Surface Sci. 83 (1979) 45.

[13] A.G.J. de Wit, R.P.N. Bronckers and J.M. Fluit, Surface Sci. 82 (1979) 177.

[14] R. van Hardeveld and A. van Montfoort, Surface Sci. 4 (1966) 396.

[15] K.R. Lawless and A.T. Gwathmey, Acta Met. 4 (1956) 153.

[16] M. Jardinier-Offergeld and F. Bouillon, in: Reaction Kinetics in Heterogeneous Chemical Systems, Ed. P. Barret (Elsevier, Amsterdam, 1975) p. 347.

[17] H. Albers, W.J.J. van der Wal, O.L.J. Gijzeman and G.A. Bootsma, Surface Sci. 77 (1978) 1 ;

H. Albers, Thesis, Univ. Utrecht (1978).

[18] K. Horn, M. Hussain and J. Pritchard, Surface Sci. 63 (1977) 244. 